\title{
Tendência temporal das internações por pneumonia em adultos e idosos na cidade do Salvador-Bahia, no período de 2003 a 2016
}

\author{
Temporal trend of hospitalizations for pneumonia in adults and elderly in the city of \\ Salvador-Bahia, from 2003 to 2016
}

\author{
Camile Xavier Souza Santos ${ }^{1}$, Adelmir Souza-Machado ${ }^{2 *}$ \\ ${ }^{1}$ Mestranda em Processos Interativos dos Órgãos e Sistemas, UFBA. \\ ${ }^{2}$ Médico. Professor Associado do Instituto de Ciências da Saúde, UFBA e do \\ Programa de Pós-Graduação em Processos Interativos dos Órgãos e Sistemas, UFBA.
}

\begin{abstract}
Resumo
Introdução: a pneumonia é uma doença infecciosa aguda do sistema respiratório que representa um grave problema de saúde pública no Brasil e no mundo. Objetivo: descrever a distribuição das internações por pneumonia em indivíduos residentes no município de Salvador, Estado da Bahia, no período de 2003 a 2016, conforme custo, sexo e faixa etária. Metodologia: estudo observacional descritivo e exploratório sobre internações por pneumonia (CID: J12-18) na cidade do Salvador, Bahia. Os dados foram obtidos no Sistema de Informações Hospitalares do Sistema Único de Saúde (SIH-SUS), disponibilizados pelo Departamento de Informação do Sistema Único de Saúde (DATASUS), tabulados e tratados no Microsoft Excel 2016. Resultados: o número de internações por pneumonia totalizou 224.173. O sexo feminino correspondeu a $51,25 \%$ das internações. Em contrapartida, o sexo masculino apresentou os maiores custos médios de internação, tendo sido o mais elevado o de R\$2.416,40 em 2016. Para ambos os sexos, as faixas etárias de maior ocorrência de internação foram as de 70-79 e a igual ou superior a 80 anos. 0 ano de 2015 apresentou a mais alta taxa de internação $(97,1 / 100$ mil hab.) e o mais alto custo médio $(\mathrm{R} \$ 2.309,61)$. Conclusão: os dados revelam declínios pontuais das internações e elevação dos custos em termos nominais. Acompanhar a evolução da morbidade em série temporal contribui para questionar mudanças de gestão pública e avaliar o impacto da doença na coletividade.
\end{abstract}

Palavras-chave: Pneumonia. Morbidade. Sistemas de Informação Hospitalar.

\begin{abstract}
Introduction: pneumonia is an acute infectious disease of the respiratory system that represents a serious public health problem in Brazil and in the world. Objective: describe the distribution of hospitalizations for pneumonia in individuals residents in the municipality of Salvador, State of Bahia, in the period from 2003 to 2016, as cost, sex and age group. Methodology: descriptive and exploratory observational study on hospitalizations for pneumonia (CID: J12-18) in the city of Salvador, Bahia. The data were obtained from the Hospital Information System of the Unified Health System (SIH-SUS), provided by the Department of Information of the Unified Health System (DATASUS), tabulated and treated in Microsoft Excel 2016. Results: the number of hospitalizations for pneumonia totaled 224,173 . Females accounted for $51.25 \%$ of hospitalizations. On the other hand, the male sex showed the highest average costs of hospitalization, and the highest was of $R \$ 2.416,40$ in 2016. For both sexes, the age groups of greater occurrence of hospitalization were those of 70-79 and the same or over 80 years of age. The year 2015 showed the highest rate of hospitalization (97.1/100 000 inhab.) and the highest average cost $(R \$ 2.309,61)$. Conclusion: the data reveal individual declines of hospitalizations and raise of costs in nominal terms. Follow the evolution of morbidity in temporal series contributes to question public management changes and assess the impact of the disease in the community.
\end{abstract}

Keywords: Pneumonia. Morbidity. Hospital Information Systems.

\section{INTRODUÇÃO}

A pneumonia é uma doença multifatorial que atinge indivíduos de todas as idades (MARTINS et al., 2002). Segundo a Organização Mundial da Saúde (2016), trata-se de uma doença infecciosa aguda do sistema respiratório cujo foco inflamatório são os alvéolos pulmonares, fundamentais para trocas gasosas, que são comumente

Correspondente/Corresponding: * Adelmir Souza-Machado - Instituto de Ciências da Saúde-Universidade Federal da Bahia - End: Av. Reitor Miguel Calmon S/N sala 413 Canela salvador-Ba CEP: 40231-300 - Tel: (71) 8875-9572 - E-mail: adelmirm@gmail.com incapacitados por agentes patogênicos ou ambientais.

A doença pode ser causada por diversos agentes infecciosos entre os quais prevalecem as bactérias, principalmente, o Streptococcus pneumoniae e o Haemophilus influenzae; os vírus, a exemplo do vírus sincicial respiratório e do influenza, e os fungos, como o Pneumocystis jiroveci (OMS, 2016; POHA, 2016). A pneumonia mais frequente é a bacteriana, que atinge principalmente crianças menores de cinco anos de idade e idosos, sendo, dentre estes últimos, mais propensos os de idade superior a 65 anos (MEDEIROS et al., 2016; MOCELIN; DOS SANTOS, 2013) 
A cada ano, cerca de 1,6 milhões de pessoas morrem por pneumonia pneumocócica no mundo (POHA, 2016). Em um estudo longitudinal, analisando-se a tendência do coeficiente de mortalidade por pneumonia nas diversas regiões do Brasil, identificou-se o crescimento do coeficiente de óbitos em 10 entre 1996 e 2012. As regiões Norte e Nordeste apresentaram uma tendência de crescimento da mortalidade ao longo dos mencionados anos, diferentemente das outras regiões, nas quais houve um decréscimo até os anos 2000 (FERRAZ; OLIVEIRA-FRIESTINO; FRANCISCO, 2017).

No Brasil, entre 2002 e 2011, foram investidos mais de $\mathrm{R} \$ 59$ bilhões em internações somente para a população adulta e idosa (SILVEIRA et al., 2013). Em Salvador, no ano de 2017, o custo total referente às internações hospitalares autorizadas por pneumonia foi de $\mathrm{R} \$ \mathbf{7} .259 .980,34$ (DATASUS, 2018c). Os avanços técnico-científicos e epidemiológicos têm permitido diagnósticos mais sofisticados e o desenvolvimento de técnicas de prevenção, viabilizando o estudo biomolecular da doença, a ponto de produzirem-se vacinas e antibióticos para tratamento (THOMSON; HARRIS, 2011). Apesar desses avanços, sendo a pneumonia uma doença oportunista, de fácil transmissão, de esclarecimento descontínuo nas comunidades de risco (MOCELIN; DOS SANTOS, 2013) e por não possuir especificidade clínica quanto à etiologia (RODRIGUES; SILVA FILHO; BUSH, 2002), vem promovendo o aumento das admissões hospitalares e dos custos para tratamento (NUNES et al., 2017).

O objetivo deste estudo é descrever as distribuições e o custo médio das internações por pneumonia em indivíduos residentes no município de Salvador, Estado da Bahia, de idade igual ou superior a 15 anos, no período de 2003 a 2016, a partir de dados disponibilizados pelo Departamento de Informação do Sistema Único de Saúde (DATASUS).

\section{METODOLOGIA}

\section{DESENHO DO ESTUDO}

Estudo epidemiológico observacional descritivo e exploratório das internações por pneumonia em indivíduos residentes no município de Salvador, Bahia. O período de análise correspondente é o de 2003 a 2016. Os dados foram obtidos a partir do Sistema de Informações Hospitalares do SUS (SIH-SUS), um banco de dados secundários de abrangência nacional disponibilizados pelo DATASUS (<http://datasus.saude.gov.br/>). Essas informações foram estratificadas pelas seguintes faixas etárias: 15 a 19 anos, 20 a 29 anos, 30 a 39 anos, 40 a 49 anos, 50 a 59 anos, 60 a 69 anos, 70 a 79 anos e 80 anos e mais. Os dados populacionais foram obtidos do Instituto Brasileiro de Geografia e Estatística (IBGE), também disponíveis no DATASUS.

\section{LOCAL DO ESTUDO}

A área estabelecida para o estudo foi a cidade do Salvador, Estado da Bahia, situada na Zona da Mata da Região Nordeste do Brasil, de clima tropical atlântico, quente úmido, com temperatura média anual em torno dos $25^{\circ} \mathrm{C}$ e precipitações abundantes durante o ano. Segundo o último censo demográfico realizado pelo IBGE (2010), a população de Salvador era de 2.675 .656 habitantes, composta, em sua grande maioria, por adultos jovens e por adultos, isto é, com idades entre 15 e 59 anos. De acordo com as estimativas para 2017, a população de Salvador seria composta por 2.953.986 habitantes. O índice de desenvolvimento humano municipal (IDHM) foi de 0,759 em 2010, e o produto interno bruto (PIB) per capita, de $\mathrm{R} \$ 19.812,07$ em 2015.

\section{ANÁLISE DOS DADOS}

Os dados para análise foram coletados no segundo trimestre de 2018. As variáveis selecionadas foram: Capítulo CID-10 (X-Doenças do aparelho respiratório); lista de morbidade CID-10 (Pneumonia: J12-J18); faixa etária (indivíduos de idade igual ou superior a 15 anos); sexo (masculino e feminino); internações por local de residência (292.740 Salvador); anos de processamento (2003-2016); valor médio de internação (custo total por internação).

Os dados obtidos foram tabulados em planilhas do software Microsoft Excel versão 2016 e passaram por um tratamento estatístico descritivo no qual foram calculadas as proporções e as variações percentuais. Para o cálculo das taxas de internação foi considerado o número de internações por população residente no período, multiplicado por 100.000 .

Para o presente estudo, não foi necessária a obtenção do termo de aprovação do Comitê de Ética em Pesquisa, visto que os dados secundários estão disponíveis para a esfera pública, não havendo qualquer restrição de acesso nem de divulgação de informações pessoais dos indivíduos.

\section{RESULTADOS}

O número de internações por pneumonia em indivíduos residentes do município de Salvador, Bahia, de idade igual ou superior a 15 anos, durante o período de 2003 a 2016, foi de 224.173. Conforme a Tabela 1, a faixa etária de maior distribuição foi a de 80 anos e mais, com 42.240 internações $(18,84 \%)$, das quais $53,25 \%$ correspondem a indivíduos do sexo feminino. Por outro lado, a faixa etária de menor distribuição foi a de 15 a 19 anos, com 16.015 internações $(7,14 \%)$, das quais $52,11 \%$ de indivíduos do sexo feminino.

O sexo feminino foi a categoria de maior proporção de indivíduos internados por pneumonia. Somente nas faixas etárias de 40 a 49 anos e de 60 a 69 anos, as internações de indivíduos do sexo masculino superaram minimamente 
as do sexo feminino $(50,39 \%$ x 49,61\% e $50,36 \%$ x 49,64\%, respectivamente).

Tabela 1 - Proporção (\%) e distribuição do número de internações por pneumonia, por faixa etária e sexo. Salvador/BA, 2003-2016.

\begin{tabular}{lcrrrrr}
\hline $\begin{array}{c}\text { Faixa etária } \\
\text { (anos) }\end{array}$ & $\mathbf{N}$ & $\mathbf{N}(\%)$ & Masculino & $\mathbf{M}(\%)$ & Feminino & $\mathbf{F}(\%)$ \\
\hline $15-19$ & 16.015 & 7,14 & 7.670 & 47,89 & 8.345 & 52,11 \\
$20-29$ & 26.861 & 11,98 & 12.632 & 47,03 & 14.229 & 52,97 \\
$30-39$ & 24.605 & 10,98 & 12.218 & 49,66 & 12.387 & 50,34 \\
$40-49$ & 24.021 & 10,72 & 12.104 & 50,39 & 11.917 & 49,61
\end{tabular}

\begin{tabular}{lllllll} 
50-59 & 26.335 & 11,75 & 13.023 & 49,45 & 13.312 & 50,55 \\
60-69 & 29.335 & 13,09 & 14.772 & 50,36 & 14.563 & 49,64 \\
$70-79$ & 34.761 & 15,51 & 17.108 & 49,22 & 17.653 & 50,78 \\
$>79$ & 42.240 & 18,84 & 19.749 & 46,75 & 22.491 & 53,25 \\
\hline Total & 224.173 & 100,00 & 109.276 & 48,75 & 114.897 & 51,25 \\
\hline \multicolumn{7}{c}{ Os valores médios totais das internações por pneu- } \\
monia somaram R\$637,35 no período de 2003 a 2007 e \\
R\$1.863,58 de 2008 a 2016. O custo mais alto evidenciou- \\
-se nas faixas etárias de 60 a 69 e de 70 a 79 anos no \\
período de 2008 a 2016. As faixas etárias de 15 a 19 e de \\
30 a 39 anos apresentaram os custos mais baixos por in- \\
ternação no período em análise (FIGURA 1). Constatou-se, \\
ainda, um aumento dos custos por internação de 2003 a \\
2015, com uma queda em 2016 (FIGURA 2).
\end{tabular}

Figura 1-Distribuição dos valores médios de internação (R\$̦) por pneumonia, por faixa etária, período e sexo. Salvador/BA, $2003-2016$.

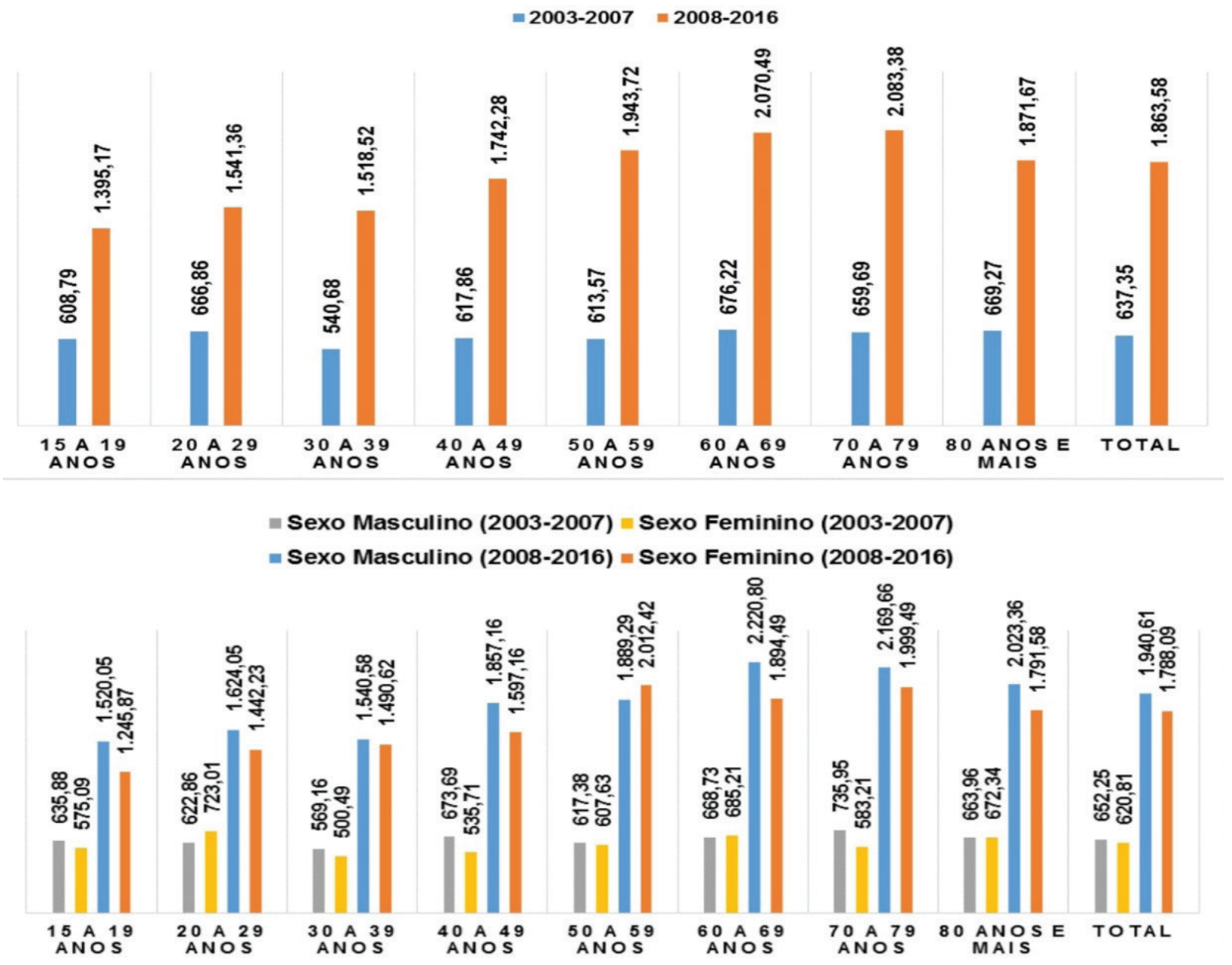

Fonte: Adaptação do DATASUS. Ministério da Saúde (2018). 
Figura 2 - Distribuição dos valores médios anuais de internação (RS) por pneumonia. Salvador/BA, 2003-2016.

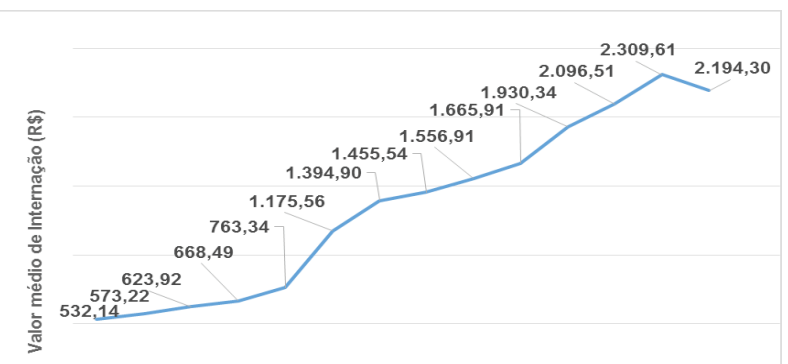

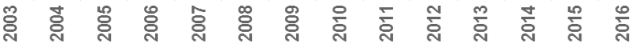

Fonte: Adaptação do DATASUS. Ministério da Saúde (2018).

Comparando-se o custo médio de internação por pneumonia do ano de 2017 para os sexos masculino e feminino ( $R \$ 2.291,04$ e $R \$ 2.322,41$, respectivamente) (DATASUS) com o custo médio dos anos de 2003 a 2016 (TABELA 2), observa-se que houve o aumento dos custos por internação e que os investimentos para os enfermos do sexo masculino foram superiores àquele para o sexo feminino. Em relação ao sexo masculino, o custo médio das internações aumentou de 2003 a 2011, sofreu uma queda em 2012, e voltou a aumentar até 2016 (R\$2.416,40), com $5,19 \%$ a mais que o custo médio do ano de 2017 . O sexo feminino, por sua vez, apresentou um aumento de 2003 a 2015, tendo-se constatado o mais alto custo médio neste último ano (R\$2.258,40), representando 2,83\% a menos que o custo médio de 2017. Entretanto, houve uma queda em 2016 (R\$1.982,95), resultando em variação de, aproximadamente, $12,20 \%$ em relação ao ano anterior.

Tabela 2 - Variação percentual (\%)* e distribuição dos valores médios de internação por pneumonia, por sexo e ano de processamento. Salvador/BA, 2003-2016.

\begin{tabular}{|c|c|c|c|c|}
\hline \multirow{2}{*}{ Ano } & \multicolumn{2}{|c|}{ Sexo masculino } & \multicolumn{2}{|c|}{ Sexo feminino } \\
\hline & $\mathrm{RS}$ & $\%$ & $\mathrm{RS}$ & $\%$ \\
\hline 2003 & 543,49 & 321,54 & 519,07 & 347,42 \\
\hline 2004 & 557,48 & 310,96 & 592,66 & 291,86 \\
\hline 2005 & 610,81 & 275,08 & 639,59 & 263,11 \\
\hline 2006 & 743,64 & 208,08 & 587,67 & 295,19 \\
\hline 2007 & 797,79 & 187,17 & 730,73 & 217,82 \\
\hline 2008 & $1.219,77$ & 87,83 & $1.129,53$ & 105,61 \\
\hline 2009 & $1.427,57$ & 60,49 & $1.359,23$ & 70,86 \\
\hline 2010 & $1.536,36$ & 49,12 & $1.373,69$ & 69,06 \\
\hline 2011 & $1.640,32$ & 39,67 & $1.475,51$ & 57,40 \\
\hline 2012 & $1.577,51$ & 45,23 & $1.748,02$ & 32,86 \\
\hline 2013 & $2.109,94$ & 8,58 & $1.756,65$ & 32,21 \\
\hline 2014 & $2.152,29$ & 6,45 & $2.041,66$ & 13,75 \\
\hline 2015 & $2.362,09$ & $-3,01$ & $2.258,40$ & 2,83 \\
\hline 2016 & $2.416,40$ & $-5,19$ & $1.982,95$ & 17,12 \\
\hline
\end{tabular}

Fonte: Adaptação do DATASUS. Ministério da Saúde (2018).
Nota: *Corresponde à diferença entre o Valor médio de internação de 2017 e o Valor médio de internação de 2003 a 2016, divida pelo Valor médio de internação de 2003 a 2016, multiplicado por 100.

A estimativa das taxas de internação por pneumonia em Salvador, durante os anos de 2003 a 2016, tendeu ao crescimento (TABELA 3). No ano de 2009, essa taxa decresceu em relação a 2008, tendo sido a mais baixa se comparada às de todos os demais anos em análise (29,0 por 100 mil hab.). Por outro lado, os anos de 2013, 2014 e 2015 apresentaram uma taxa superior a 90 internações por 100 mil habitantes (92,0; 93,9; e 97,1, respectivamente), seguindo-se a taxa de 80 internações por 100 mil habitantes $(80,6)$ obtida no ano de 2012.

Levando-se em conta a variável faixa etária (TABELA 3), os indivíduos de idade igual ou superior a 80 anos apresentaram a mais alta taxa de internação do período em análise (1.031,6/100 mil hab.).

Tabela 3 - Taxa de internação e variação percentual das internações por pneumonia, por ano de processamento, faixa etária e sexo. Salvador/BA, 2003-2016.

\begin{tabular}{|c|c|c|c|}
\hline Variáveis & Total & $\begin{array}{c}\text { Taxa de } \\
\text { internação }\end{array}$ & $\begin{array}{l}\text { Variação } \\
\text { percentual }\end{array}$ \\
\hline \multicolumn{4}{|c|}{$\begin{array}{c}\text { Ano de } \\
\text { processamento }\end{array}$} \\
\hline 2003 & 829 & 43,9 & 121 \\
\hline 2004 & 903 & 47,2 & 106 \\
\hline 2005 & 867 & 43,9 & 121 \\
\hline 2006 & 907 & 45,2 & 115 \\
\hline 2007 & 1.020 & 48,8 & 99 \\
\hline 2008 & 1.043 & 46,4 & 109 \\
\hline 2009 & 870 & 38,0 & 156 \\
\hline 2010 & 944 & 44,5 & 118 \\
\hline 2011 & 1.478 & 69,2 & 40 \\
\hline 2012 & 1.734 & 80,6 & 20 \\
\hline 2013 & 2.093 & 92,0 & 6 \\
\hline 2014 & 2.164 & 93,9 & 3 \\
\hline 2015 & 2.262 & 97,1 & 0 \\
\hline 2016 & 1.977 & $-*$ & \\
\hline \multicolumn{4}{|c|}{ Faixa etária (anos) } \\
\hline $15-19$ & 678 & 20,6 & 4.906 \\
\hline $20-29$ & 1.562 & 21,9 & 4.602 \\
\hline $30-39$ & 1.861 & 29,0 & 3.461 \\
\hline $40-49$ & 2.150 & 45,4 & 2.174 \\
\hline $50-59$ & 2.581 & 81,2 & 1.170 \\
\hline $60-69$ & 2.911 & 169,1 & 510 \\
\hline $70-79$ & 3.245 & 375,6 & 175 \\
\hline$>79$ & 4.103 & $1.031,6$ & 0 \\
\hline \multicolumn{4}{|l|}{ Sexo } \\
\hline Masculino & 109.276 & 394,0 & 5 \\
\hline Feminino & 114.897 & 414,3 & 0 \\
\hline
\end{tabular}

Fonte: Adaptação do DATASUS. Ministério da Saúde, 2018. Nota: *Estimativa populacional por faixa etária não disponibilizada pelo IBGE. 
Comparando-se as estimativas das taxas de internação por faixa etária dos anos 2012 e 2015 (TABELA 4), constata-se um decréscimo nas faixas etárias de 15 a 19 , de 20 a 29 e de 30 a 39 anos. No mesmo período, observa-se, contudo, um aumento nas faixas etárias de 60 a 69, de 70 a 79, e na faixa igual ou superior a 80 anos.

Tabela 4 - Taxa de internação e distribuição das internações por pneumonia, por faixa etária e ano de processamento. Salvador/ $B A, 2012-2015$.

\begin{tabular}{crrrrrrrrr}
\hline & \multicolumn{7}{c}{ Faixa etária (anos) } \\
\cline { 2 - 10 } & $\mathbf{1 5 - 1 9}$ & $\mathbf{2 0 - 2 9}$ & $\mathbf{3 0 - 3 9}$ & $\mathbf{4 0 - 4 9}$ & $\mathbf{5 0 - 5 9}$ & $\mathbf{6 0 - 6 9}$ & $\mathbf{7 0 - 7 9}$ & $\mathbf{7 7 9}$ & Total \\
\hline $\mathbf{2 0 1 2}$ & 92 & 138 & 166 & 210 & 274 & $\mathbf{2 5 6}$ & $\mathbf{2 7 5}$ & 323 & 1.734 \\
\hline Taxa de internação & 41,9 & 25,3 & 34,1 & 55,3 & 101,8 & 180,1 & 377,2 & 899,9 & 80,6 \\
\hline $\mathbf{2 0 1 3}$ & 65 & 142 & 197 & 238 & 295 & 321 & 358 & 477 & 2.093 \\
\hline Taxa de internação & 29,0 & 27,5 & 34,9 & 59,3 & 98,4 & 198,3 & 476,1 & 1414,2 & 92,0 \\
\hline $\mathbf{2 0 1 4}$ & 58 & 140 & 194 & 207 & 292 & 333 & 416 & 524 & 2.164 \\
\hline Taxa de internação & 25,7 & 28,0 & 33,4 & 51,1 & 94,0 & 194,5 & 535,9 & 1481,0 & 93,9 \\
\hline $\mathbf{2 0 1 5}$ & 61 & 106 & 157 & 187 & 268 & 403 & 471 & 609 & 2.262 \\
\hline Taxa de internação & 26,7 & 22,1 & 26,5 & 45,5 & 83,8 & 221,7 & 588,7 & 1652,2 & 97,1 \\
\hline
\end{tabular}

Fonte: Adaptação do DATASUS. Ministério da Saúde (2018).

\section{DISCUSSÃO}

O presente estudo objetivou descrever o comportamento da distribuição das internações por pneumonia no município de Salvador, Bahia, em indivíduos de idade igual ou superior a 15 anos, conforme sexo e custo médio por internação, durante os anos de 2003 a 2016. Verificou-se o maior número de internações em indivíduos do sexo feminino, assim como em indivíduos de idade igual ou superior a 80 anos. Observou-se o aumento do custo médio por internação de 2003 a 2015, sendo este último o de mais alto valor. Em quase todos os 14 anos sob análise, o custo médio mais alto correspondeu ao sexo masculino. As taxas aumentaram a partir do ano de 2012, alcançando mais de 60 internações por 100 mil habitantes, com redução, porém, para as faixas etárias compreendidas entre 15 e 59 anos entre 2012 e 2015.

A população de Salvador tende para o envelhecimento em vista da redução da fecundidade e das melhorias na expectativa de vida. Comparados os dados dos censos de 2000 e 2010, segundo pesquisa realizada pelo Programa das Nações Unidas para o Desenvolvimento (PNUD), pelo Instituto de Pesquisa Econômica Aplicada (IPEA) e pela Fundação João Pinheiro (FJP) (2013), constatou-se o aumento da população potencialmente ativa, de 15 a 64 anos de idade, seguido da redução da população dependente, isto é, os indivíduos menores de 15 anos.

Vasconcelos e Gomes (2012) analisaram as taxas de natalidade, de fecundidade e de mortalidade das cinco regiões brasileiras durante os anos de 1950 a 2010 e observaram que o índice de envelhecimento da sociedade brasileira aumentou de $10,3 \%$ para $44,8 \%$. No Nordeste, entre 1991 e 2010, houve uma queda na taxa de mortalidade infantil e, em 2010, o índice de envelhecimento atingiu $38,7 \%$.

Em adolescentes e adultos, as complicações por pneumonia são, muitas vezes, mais benignas se comparadas com as das populações das faixas etárias mais extremas, a dos idosos e a das crianças. Indivíduos de 50 anos ou mais estão sujeitos ao aparecimento de múltiplas doenças crônicas, como doenças cardiovasculares, renais, pulmonares, hepáticas e metabólicas, que sensibilizam o sistema imunológico, propiciando o contágio frequente e, por vezes, dificultando o tratamento, em vista de sinais e sintomas que mascaram o diagnóstico, e contribuindo para um atendimento tardio e internação já em estado grave de pneumonia (CORRÊA et al., 2009).

No período em análise, não foi identificada grande diferença para internações entre homens e mulheres. Um estudo realizado por Cardoso e Oliveira (2011) abrangendo o período de 1998 a 2007 estimou que as taxas de morbidade por pneumonia, segundo o sexo, foram mais expressivas entre homens na região metropolitana de Salvador, tendo alcançado, em 2007, para o sexo masculino, o percentual de $89,61 \%$ de internações por 100.000 habitantes, e o de $75,80 \%$ de internações por 100.000 habitantes para o sexo feminino. Silva et al. (2006) constataram, também para Salvador, um elevado risco de internamentos e de óbitos entre 1995 e 2004 para indivíduos do sexo masculino com idade superior a 56 anos.

Adultos maiores de 50 anos e idosos, por apresentarem maiores incapacidades e estarem mais propensas a morbidades, estão mais sujeitos ao uso dos produtos e dos serviços de saúde. Alguns dos motivos que intensificam a procura decorrem, muitas vezes, da falta de serviços ambulatoriais ou domiciliares, ou do desconhecimento dos fatores que promovem a exacerbação da doença respiratória, favorecendo a descoberta tardia da enfermidade e a hospitalização em estado grave, gerando elevados custos para o tratamento (DUTRA; PEREIRA, 2010).

No presente estudo, constatou-se, para indivíduos de idade superior a 59 anos, o aumento do custo médio entre os anos de 2008 e 2016, tendo sido mais elevado em indivíduos do sexo masculino. Os custos médios correspondem aos gastos médios de recursos pagos pelo SUS na prestação de atendimentos hospitalares, que compreendem serviços profissionais, materiais hospi- 
talares, medicamentos, exames, alimentação, estadia e variam de região para região na dependência de fatores socioeconômicos, demográficos e epidemiológicos (NUNES et al., 2017).

No Brasil, entre 2002 e 2011, ocorreram mais de 20 milhões de internações hospitalares entre idosos no âmbito do SUS, tendo-se despendido mais de R\$ 21 bilhões (SILVEIRA et al., 2013). Segundo o mesmo estudo, há uma variação entre o custo médio das internações entre adultos (20-56 anos) e idosos (60 anos ou mais) e, para doenças respiratórias, o custo médio para a população adulta correspondeu a $R \$ 606,22$, contra $R \$ 667,19$ entre os idosos, tendo sido mais expressivo o gasto entre as mulheres do que entre os homens.

Ainda segundo Silveira et al. (2013), observando-se questões culturais associadas ao gênero, verifica-se que muitos homens não têm o hábito de cuidar da saúde e, quando buscam assistência médica, o diagnóstico é tardio e a enfermidade já se encontra em estado avançado, requerendo exames mais específicos e tratamentos mais dispendiosos. Assim, determinantes sociais e demográficos são fatores importantes para a resolução dos índices de morbidade (ANTUNES et al., 2014; KERNKAMP et al., 2016) contudo, não foram totalmente contemplados na presente pesquisa. Segundo Antunes et al. (2014) em Salvador, a renda e o número de postos e centros de saúde tiveram relevante influência nas hospitalizações por pneumonia, entre 2001 e 2007.

O aumento das internações com posterior queda em 2016, como visto para o presente estudo, sugere, possivelmente, resultados positivos provenientes das campanhas de prevenção (LUNA; GATTÁS; CAMPOS, 2014), da ampliação da cobertura vacinal nos anos anteriores (DATASUS, 2018b) e do monitoramento de sorotipos, permitindo atestar grupos de ocorrências, desenvolver novas vacinas e melhorar as já existentes (MEDEIROS et al., 2016). Pode-se supor que o aumento das internações após 2012 deva-se ao número de leitos ativos (DATASUS, 2018a), ao sorotipo do patógeno (MEDEIROS et al., 2016; RODRIGUES; SILVA FILHO; BUSH, 2002), a fatores climáticos e ambientais (MARTINS et al., 2002; SILVA et al., 2006) e a transições socioeconômicas e demográficas (VASCONCELOS; GOMES, 2012) vigentes à época.

Cabem algumas observações sobre a realização do tipo de estudo aqui apresentado em que não são contemplados dados de hospitais não conveniados ao SUS, gerando o chamado viés de seleção ao promover uma representação ilusória de uma parcela da população economicamente mais favorecida (TOYOSHIMA; ITO; GOUVEIA, 2005).

O SIH-SUS registra todas as informações que derivam das internações hospitalares financiadas pelo SUS (DATASUS, 2018d). Seu banco de dados abarca milhões de internações, todos os anos, que são úteis para o planejamento, as transferências financeiras, a gestão dos serviços de saúde e as investigações epidemiológicas e de vigilância sanitária (DATASUS, 2018d; VERAS; MARTINS,
1994). Sua confiabilidade, ou seja, a igualdade informacional entre os dados dos prontuários e o que é fornecido no portal já ficou comprovada (VERAS; MARTINS, 1994). O treinamento do corpo técnico, a uniformização das plataformas e os avanços na informática (DATASUS, 2018d) viabilizam o melhoramento do conteúdo disponibilizado e sua fidedignidade à realidade, sendo, desse modo, amplamente utilizado por diversas pesquisas (ANTUNES et al., 2014; CARDOSO; OLIVEIRA, 2011; DUTRA; PEREIRA, 2010; FERRAZ; OLIVEIRA-FRIESTINO; FRANCISCO, 2017).

\section{CONCLUSÃO}

O presente estudo revelou que o número de internações por pneumonia em indivíduos residentes em Salvador, Bahia, aumentou entre 2003 e 2015, mas com declínios pontuais. $O$ sexo masculino apresentou os custos mais elevados por internação, e o sexo feminino, o maior número de internações. As faixas etárias de maior ocorrência foram a de 70-79 anos e a superior a 79 anos, para as quais também se constataram os mais altos valores médios de internação.

A alta prevalência de internações por pneumonia em adultos (20-59 anos) e em idosos (60 anos ou mais) em Salvador faz repensar a estrutura sociodemográfica e econômica da região, bem como pode motivar possíveis mudanças nas estratégias de prevenção e de tratamento na rede pública, que devem abranger, principalmente, a população idosa.

Descrever como se distribuem as internações em uma série temporal permite acompanhar a evolução da morbidade e formular questionamentos que contribuam para avaliar mudanças de gestão pública em um município e o impacto da doença na coletividade.

\section{REFERÊNCIAS}

ANTUNES, F. P. et al. Social determinants of intra-urban differentials of admissions by respiratory diseases in Salvador (BA), Brazil. Rev. bras. epidemiol., São Paulo, v. 17, supl. 2, p. 29-38, 2014.

CARDOSO, J. P.; OLIVEIRA, M. N. D. DE. Tendência temporal das doenças respiratórias no município de Salvador. Rev. bras. promoç. saúde, Fortaleza, v. 24, n. 1, p. 31-39, 30 mar. 2011.

CORRÊA, R. DE A. et al. Diretrizes brasileiras para pneumonia adquirida na comunidade em adultos imunocompetentes -2009 . J. bras. Pneumol., Brasília, v. 35, n. 6, p. 574-601, jun. 2009.

DATASUS. Ministério da Saúde. CNES, recursos físicos, hospitalar, leitos de internação, Bahia: Salvador. 2018a. Disponível em: <http://tabnet. datasus.gov.br/cgi/tabcgi.exe?cnes/cnv/leiintba.def>. Acesso em: 7 ago. 2018.

DATASUS. Ministério da Saúde. Imunizações, Cobertura, Bahia: Salvador. 2018b. Disponível em: <http://tabnet.datasus.gov.br/cgi/tabcgi.exe?pni/ CNV/CPNIBA.def>. Acesso em: 7 ago. 2018.

DATASUS. Ministério da Saúde. Morbidade hospitalar do SUS, por local de residência, Bahia: Salvador. 2018c. Disponível em: <http://tabnet.datasus.gov.br/cgi/tabcgi.exe?sih/cnv/nrba.def>. Acesso em: 7 ago. 2018.

DATASUS. Ministério da Saúde. SIHSUS - Sistema de informações hospitalares do SUS. 2018d. Disponível em: <http://datasus.saude.gov. 
br/sistemas-e-aplicativos/hospitalares/sihsus>. Acesso em: 5 jul. 2018.

DUTRA, G. F.; PEREIRA, A. M. Temporal analysis of hospital admission and deaths by respiratory diseases among elderly people, Minas Gerais state, Brazil. Rev. bras. geriatr. gerotol., Rio de Janeiro, v. 13, n. 1, p. 12, 2010.

FERRAZ, R. DE O.; OLIVEIRA-FRIESTINO, J. K.; FRANCISCO, P. M. S. B. Pneumonia mortality trends in all Brazilian geographical regions between 1996 and 2012. J. bras. pneumol., Brasília, v. 43, n. 4, p. 274-279, Aug. 2017.

IBGE. Instituto Brasileiro de Geografia e Estatística: Brasil em síntese, Bahia, Salvador. 2010. Disponível em: < https://cidades.ibge.gov.br/ brasil/ba/salvador/panorama>. Acesso em: 8 ago. 2018.

KERNKAMP, C. DA L. et al. Perfil de morbidade e gastos hospitalares com idosos no Paraná, Brasil, entre 2008 e 2012. Cad. Saúde Pública, Rio de Janeiro, v. 32, n. 7, 2016.

LUNA, E. J. DE A.; GATTÁS, V. L.; CAMPOS, S. R. DE S. L. DA C. Efetividade da estratégia brasileira de vacinação contra influenza: uma revisão sistemática. Epidemiol. serv. saúde, Brasília, v. 23, n. 3, p. 559-576, set. 2014.

MARTINS, L. C. et al. Poluição atmosférica e atendimentos por pneumonia e gripe em São Paulo, Brasil. Rev. Saúde Pública, São Paulo, v. 36, n. 1, p. 88-94, fev. 2002.

MEDEIROS, M. I. C. et al. Clinical and microbiological implications of invasive pneumococcal disease in hospitalized patients (1998-2013). Braz J. Infect. Dis., Salvador, v. 20, n. 3, p. 242-249, May 2016.

MOCELIN, C. A.; DOS SANTOS, R. P. Community-acquired pneumonia at the Hospital de Clínicas de Porto Alegre: evaluation of a care protocol. Braz J. Infect. Dis., Salvador, v. 17, n. 5, p. 511-515, Sept. 2013.

NUNES, S. E. A. et al. Hospitalization costs of severe bacterial pneumonia in children: comparative analysis considering different costing methods. Einstein (São Paulo), São Paulo, v. 15, n. 2, p. 212-219, June 2017.

ORgANIZAÇÃO MUNDIAL DA SAÚDE (OMS). Pneumonia. 2016. Dis- ponível em: <http://www.who.int/news-room/fact-sheets/detail/ pneumonia>. Acesso em: 7 ago. 2018.

PNUD; IPEA; FJP. Atlas do desenvolvimento humano no Brasil - Salvador, Ba. 2013. Disponível em: <http://www.atlasbrasil.org.br/2013/pt/ perfil_m/3225>. Acesso em: 8 jun. 2018.

PANAMERICAN HEALTH ORGANIZATION (POHA). Clinical aspects of bacterial pneumonia. 2016. Disponível em: <https://www.paho.org/ hq/index.php?option=com_content $\&$ view $=$ article $\&$ id $=1896 \% 3$ A2009-clinical-aspects-bacterial-pneumonia\&catid=1591\%3Aabout\&Itemid $=0 \&$ lang=en $>$. Acesso em: 7 ago. 2018.

RODRIGUES, J. C.; SILVA FILHO, L. V. F. da; BUSH, A. Diagnóstico etiológico das pneumonias: uma visão crítica. J. pediatr., Rio de Janeiro, v. 78, supl. 2, p. 129-140, 2002.

SILVA, B. M. P. da et al. Tendência da morbimortalidade por pneumonia na região metropolitana de Salvador - 1998 a 2004. Rev. baiana saúde pública, Salvador, v. 30, n. 2, p. 294-308, 1 dez. 2006.

SILVEIRA, R. E. DA et al. Gastos relacionados a hospitalizações de idosos no Brasil: perspectivas de uma década. Einstein (São Paulo), São Paulo, v. 11, n. 4, p. 514-520, dez. 2013.

THOMSON, A.; HARRIS, M. Community-acquired pneumonia in children: what's new? Thorax, London, v. 66, n. 10, p. 927-928, 1 Oct. 2011.

TOYOSHIMA, M. T. K.; ITO, G. M.; GOUVEIA, N. Morbidade por doenças respiratórias em pacientes hospitalizados em São Paulo/SP. Rev. Assoc. Med. Bras., São Paulo, v. 51, n. 4, p. 209-213, ago. 2005.

VASCONCELOS, A. M. N.; GOMES, M. M. F. Transição demográfica: a experiência brasileira. Epidemiol. serv. saúde, São Paulo, v. 21, n. 4, p. 539-548, dez. 2012.

VERAS, C. M. T.; MARTINS, M. S. A confiabilidade dos dados nos formulários de Autorização de Internação Hospitalar (AIH), Rio de Janeiro, Brasil. Cad. Saúde Pública, Rio de Janeiro, v. 10, n. 3, p. 339-355, set. 1994.

Submetido em: $14 / 11 / 2018$

Aceito em: 29/11/2018 